\title{
Geometrical Properties of Coronal Mass Ejections
}

\author{
Hebe Cremades ${ }^{1}$ and Volker Bothmer ${ }^{1}$ \\ ${ }^{1}$ Max-Planck-Institut für Sonnensystemforschung, Katlenburg-Lindau, D 37191, Germany \\ email: cremades@mps.mpg.de
}

\begin{abstract}
Based on the SOHO/LASCO dataset, a collection of "structured" coronal mass ejections (CMEs) has been compiled within the period 1996-2002, in order to analyze their threedimensional configuration. These CME events exhibit white-light fine structures, likely indicative of their possible 3D topology. From a detailed investigation of the associated low coronal and photospheric source regions, a generic scheme has been deduced, which considers the whitelight topology of a CME projected in the plane of the sky as being primarily dependent on the orientation and position of the source region's neutral line on the solar disk. The obtained results imply that structured CMEs are essentially organized along a symmetry axis, in a cylindrical manner. The measured dimensions of the cylinder's base and length yield a ratio of 1.6. These CMEs seem to be better approximated by elliptic cones, rather than by the classical ice cream cone, characterized by a circular cross section.
\end{abstract}

Keywords. Sun: corona, Sun: coronal mass ejections (CMEs), Sun: prominences

\section{Introduction}

Coronal Mass Ejections (CMEs) constitute magnificent explosive events observed in the solar corona (e.g. Munro et al. 1979; Hundhausen et al. 1984). They were discovered by space-borne coronagraphs. Coronagraphs record the density of free electrons in the coronal plasma, integrated along the line of sight. The two-dimensional nature of coronagraphic images as well as the current restriction to single viewpoint observations constrain our view and knowledge regarding the three-dimensional configuration and geometry of CMEs. In the earliest studies it had been proposed that CMEs may be planar looplike structures (e.g. Trottet \& MacQueen 1980). On the contrary, other studies supported rather a shell-like configuration (e.g. Webb 1998). CMEs are presently known as threedimensional structures seen in projection on the plane of the sky. However, it is still a subject of debate whether the 3D structure resembles more an arcade or a spherical bubble.

Cremades \& Bothmer (2004) studied a set of 276 structured CMEs selected from the full set of LASCO (Large Angle Spectroscopic Coronagraph) C2 images during the period 1996-2002, in order to gain information about the three-dimensional configuration of CMEs. Their associated source regions in the low corona and photosphere could be uniquely identified in 124 of the cases. These were located essentially in limb and nearlimb regions, as expected from the clarity of the observed (projected) CME profile and direction of propagation. From the analysis of the structured CMEs and their associated source regions, based on the comparison of magnetic structures in the low corona as recorded by the Extreme UV Imaging Telescope (EIT) with the profile of the structured white light CME as observed by the LASCO coronagraphs, a generic scheme for CME configurations was deduced. It basically considers the structured CMEs as cylindrical entities (as supported by the later study of Moran \& Davila 2004), whose projected 


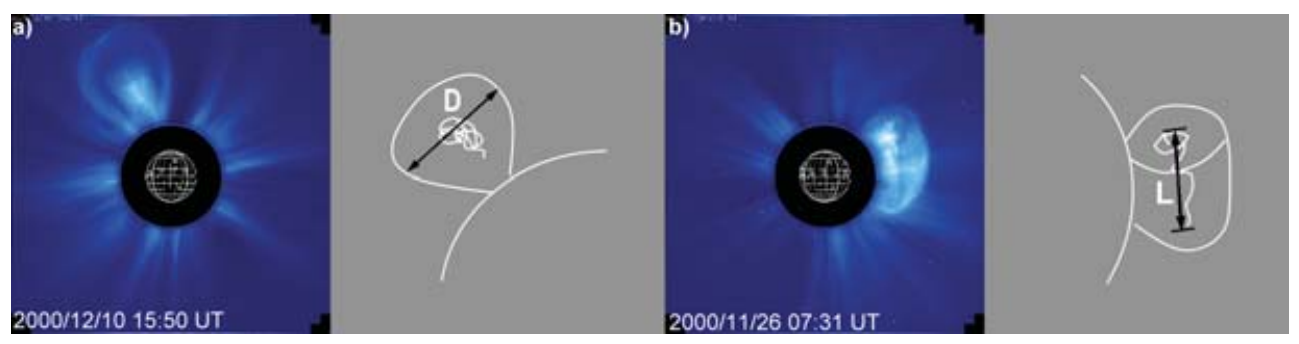

Figure 1. Examples of two CMEs seen in extreme projection: a) main axis primarily oriented along the line of sight, i.e. CME seen in cross-section with diameter $D$; b) main axis $L$ oriented perpendicular to the line of sight.

profiles depend on the neutral line locations and orientations, consistent with the axes of the approximately cylindrically-symmetric CMEs.

In the next section we present width measurements for the subset of structured CMEs that exhibited extreme projections: seen approximately along their axis, or perpendicular to it. These two groups of projection yielded average widths rather distinct, revealing an asymmetry between the axial direction and the perpendicular one. In Sections 3 and 4 we attempt to find the same discrepancy in halo CMEs, via an elliptical cone model based on the circular cone model introduced by Zhao et al. (2002).

\section{Typical Dimensions of cylindrically shaped CMEs}

The high order of cylindrical symmetry evident from the analyses of the 124 structured CMEs with associated source regions listed in Cremades \& Bothmer (2004) raises the question of whether the angular widths (AWs) of CMEs seen along their symmetry axis differ from those seen perpendicularly to it (see Fig. 1). In order to investigate the degree of similarity in the AWs of these two views, the structured CMEs exhibiting the two purest cases of projection -seen along their axis or perpendicular to it- were identified from the set of 124 structured CMEs. Examples of the two cases of projection are depicted in Fig. 1. Their AWs were measured in LASCO/C2 and, if the (leading edge was visible) in EIT $195 \AA$ images, in EIT as well. Altogether we identified 16 events seen along their axis, out of which 9 were also measurable in EIT $195 \AA$; and 17 events seen perpendicular to their axis, out of which 9 were measurable in EIT $195 \AA$. The angular width " $D$ " of the axial cases indicates the diameter of the imaginary cylinder's cross-section (Fig. 1a), while the width " $L$ " of the events observed perpendicular to their axis denotes the length of the imaginary cylinder's axis (Fig. 1b), most likely aligned with the extended prominence material.

The AWs derived from the analyzed events were put together in distribution histograms for $D$ and $L$ as shown in Fig. 2. Each histogram shows in different colors the values measured in the LASCO/C2 and EIT $195 \AA$ fields of view. Although limb CMEs seem to keep their AWs in the field of view of $\mathrm{LASCO} / \mathrm{C} 2$ and $\mathrm{C} 3$, there is a marked difference between the widths measured in EIT and in $\mathrm{LASCO} / \mathrm{C} 2$, supporting the assumption that the CME's greatest expansion takes place already between 1 and 2.2 solar radii, i.e. into the region of transition from open to closed coronal fields. The averages of $D$ in EIT and in $\mathrm{LASCO} / \mathrm{C} 2$ were $14^{\circ}$ and $37^{\circ}$, measured at average heights of 1.3 and 4 solar radii, respectively. The larger averages of $L$ in EIT and in $\mathrm{LASCO} / \mathrm{C} 2$ yielded $22^{\circ}$ and $58^{\circ}$ respectively, measured at identical heights. The ratio $L / D$, considered with the purpose of comparing the average $D$ and $L$ of the imaginary cylinder, yielded the value of 1.6 for both EIT and LASCO/C2 data. That the ratio does not change from the EIT to the 

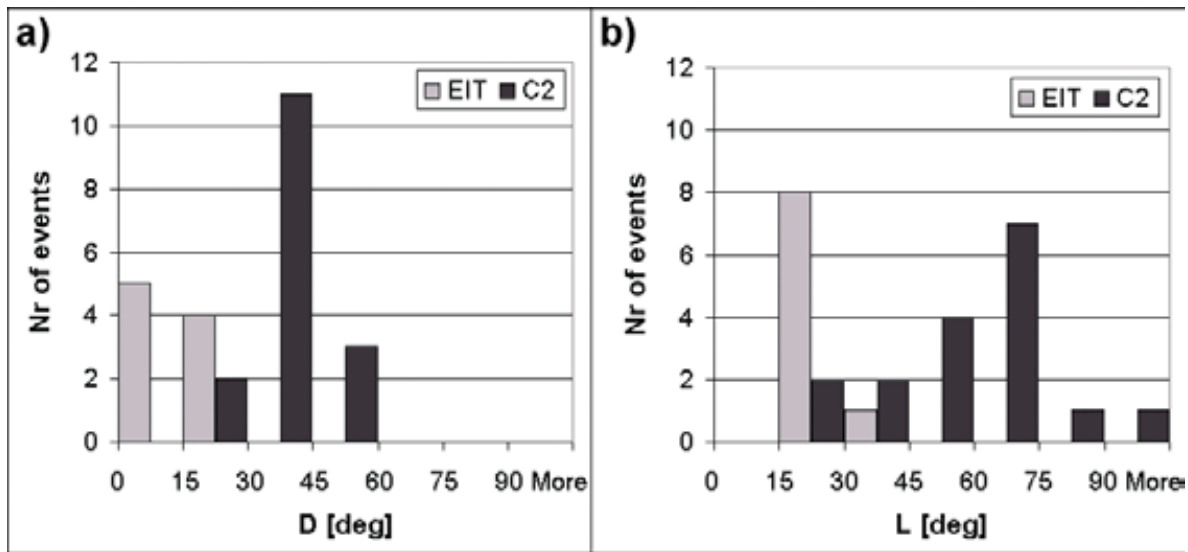

Figure 2. AW distribution histogram for the structured CMEs seen in extreme projection. Measurements carried out in EIT $195 \AA$ are shown in light grey, and those in LASCO/C2 in dark grey. a) Main axis primarily oriented along the line of sight, b) main axis oriented perpendicular to the line of sight.

LASCO/C2 field of view for this particular set of events can be interpreted as a sign of self-similarity expansion, as addressed by some theoretical models (e.g. Gibson \& Low 2000).

The arcade-like rather than bubble-like structure derived from the basic CME scheme implies an essential difference in the profiles projected in the plane of the sky, depending on the arcade's orientation with respect to the observer. If the arcade is approximated by a cylinder, the measured widths of its cross-section $D$ and length $L$ can be substantially different. It is expected that halo CMEs will present cases of asymmetry as well, as a consequence of the imprints of the source region characteristics. Halos entirely seen from top, i.e. originating from exactly the center of the solar disk, should not look circular, but rather elliptical. On the other hand, the presence of elliptical full halos in LASCO/C2/C3 observations could merely be a result of projection effects, i.e. of intrinsically circular halo CMEs traveling in other directions than exactly the line of sight.

The circular cone model introduced by Zhao et al. (2002) approximates CMEs as cones with circular base and allows the user to observe the base of this cone as projected on the plane of the sky for events propagating in different directions, apart from the line of sight. The result of projecting the base of a circular cone on the plane of the sky is typically an ellipse. Though after the implementation of the circular cone model from Zhao et al. (2002) still a substantial number of events could not be fitted, suggesting the existence of real elliptical halos, observed as such not only as an effect of projection. In order to prove the existence of asymmetric halos, an elliptical cone model was developed, in an effort to reproduce fairly well a set of halo CMEs observed by LASCO.

\section{The elliptical cone model}

The elliptical cone model was developed on the basis of the circular cone model presented by Zhao et al. (2002), assuming as well radial propagation and constant AWs. To generate an elliptical cone, not only one half-AW of the cone is needed, but two: one that grows in the direction of the semi minor axis and another that grows in the direction of the semi major axis, called $\omega_{a}$ and $\omega_{b}$ respectively (see Fig. 3). From these two half-AWs arise the two slant heights $s_{a}$ and $s_{b}$, which are related by the condition $s_{a} \cos \left(\omega_{a}\right)=s_{b} \cos \left(\omega_{b}\right)$. The fact of producing an elliptical base involved the insertion of 


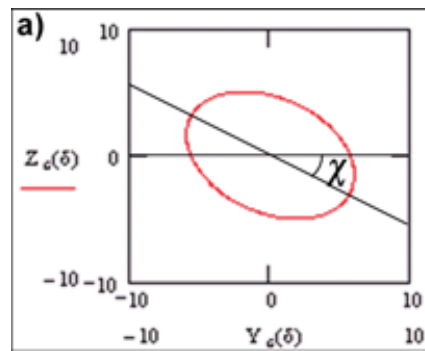

b)

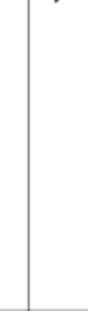

c)

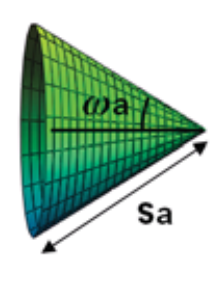

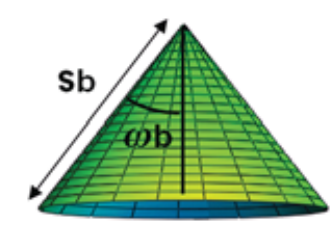

Figure 3. The three views of the elliptic cone. a) Plane $Z_{c}-Y_{c}$ : the base of the cone. b) Plane $Z_{c}-X_{c}$ : minor width of the cone. c) Plane $Y_{c}-X_{c}$ : major width of the cone.

a new variable $\chi$ that defines the tilt of the semimajor axis with respect to the horizontal. Then the equations that define the cone in its own coordinate system are given by:

$$
\begin{gathered}
X_{c}(\delta)=s_{a} \cos \left(\omega_{a}\right) \\
Y_{c}(\delta)=s_{b} \sin \left(\omega_{b}\right) \cos (\delta) \cos (\chi)+s_{a} \sin \left(\omega_{a}\right) \sin (\delta) \sin (\chi) \\
Z_{c}(\delta)=s_{a} \sin \left(\omega_{a}\right) \sin (\delta) \cos (\chi)-s_{b} \sin \left(\omega_{b}\right) \cos (\delta) \sin (\chi)
\end{gathered}
$$

Where, using the same nomenclature of the circular cone model when applicable, $X_{c}$ is directed along the central axis of the cone, $Y_{c}$ and $Z_{c}$ are oriented in the directions of the semi major and semi minor axis respectively (whenever tilt $\chi=0$ ), and $\delta$ is the parameter that varies from $0^{\circ}$ to $360^{\circ}$. The three different views of the cone are shown in Fig. 3 .

To project the base's rim of the elliptical cone against the plane of the sky, the same transformation as applied by Zhao et al. (2002) was employed, by means of the heliographic latitude $\lambda$ and longitude $\phi$ in which the central axis of the cone is oriented.

A system of six equations with six unknowns arises after matching the parametric equation of the ellipse measured in the LASCO/C2/C3 images and that of the ellipse synthetically generated by the model (representing the rim of the cone's base projected on the plane of the sky); making it possible to find analytical solutions for all the variables $\omega_{a}, \omega_{b}, s_{a}, s_{b}, \lambda, \phi$ and $\chi$. Unfortunately, due to the fact of dealing with transcendent equations, the solution is not unique though constrained to four equivalent ranges of noncomplex values, depending on the location of the start point of the ellipse. The central solution of the value range has been established as the most probable one, as a tradeoff solution for all cases.

\section{Application to halo CMEs}

In an effort to test whether the ratio $L / D$ also compares to measurements of halo CMEs, the elliptical cone model was applied to a set of halo CMEs, selected from the halo database at ftp://ares.nrl.navy.mil/pub/lasco/halo. The 32 selected events were characterized for being full halos, front sided and very bright. The latter attribute was preferred because of the simplicity while choosing the boundary that defined the ellipse to be approximated by the model. To construct the ellipse that best approximated each full halo CME, the coordinates of five points that defined the ellipse were determined in the image of the sequence that showed the sharpest leading edge (see an example in Fig. 4). The parametric equations of the so obtained ellipse were matched with those of the projected cross section of an elliptical cone, obtaining in this way its characteristic geometric parameters. Although these geometric parameters are not unique, they have been calculated for all cases under the same conditions, which allows for comparisons between them. 


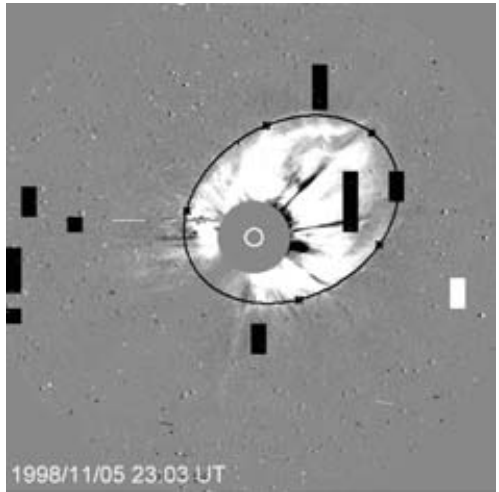

Figure 4. Running-difference image of a bright full halo recorded on November 5th 1998 by the LASCO/C3 coronagraph. The thick black line marks the fitted ellipse and the crosses the five points from which the ellipse was derived.

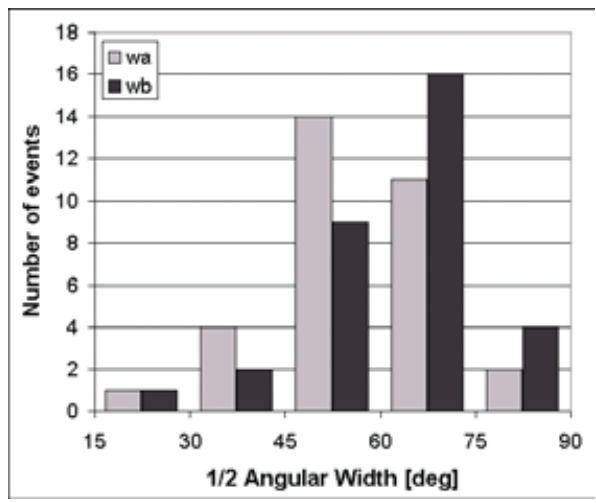

Figure 5. Half-AW distribution histogram for the analyzed halo CMEs. Light grey columns correspond to half-AWs in the direction of minor width $\left(\omega_{a}\right)$, while dark grey columns refer to half-AWs in the direction of major width $\left(\omega_{b}\right)$.

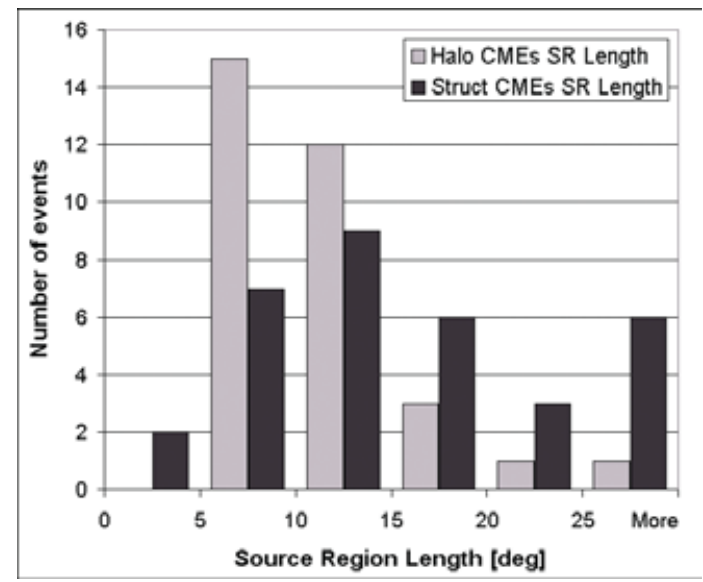

Figure 6. Distribution histogram for the source region lengths of both sets of analyzed CMEs.

The distribution histogram of the AWs $\omega_{a}$ and $\omega_{b}$ as measured in the semi minor and semi major directions (see Fig. 5) indicates that the asymmetry does also exist in halo CMEs, due to the shift visible in the two distributions for this set of 32 halo CMEs. The average ratio $\left.<\omega_{b} / \omega_{a}\right\rangle=1.11$ is though not so remarkable when compared with the value $\langle L\rangle /\langle D\rangle=1.6$ calculated from the subset of structured CMEs having extreme projections.

The reason for the discrepancy in the symmetry ratio for the compared groups of CMEs (structured and halo) may lie in the different features that characterize both groups of events. The findings from Cremades \& Bothmer (2004) suggest that the degree of cylindrical symmetry depends on the length of the neutral line that is associated with the CME origins. Fig. 6 displays the lengths of the associated source regions for both sets of CMEs. The distribution for the analyzed halo CMEs is narrower, with the average located at $11^{\circ}$; while that of the structured CMEs is broader, reaching the $40^{\circ}$, with the average located at $16^{\circ}$. The dissimilitude in the length distributions likely explains the discrepancies found in the two sets of CMEs. 


\section{Summary and conclusions}

We have investigated a subset of structured CMEs analyzed in a previous study by Cremades \& Bothmer (2004), characterized by the alignment of the main axis of symmetry either primarily along the line of sight or perpendicular to it. The average width of structured CMEs with the axis oriented along the line of sight $(D)$ yielded $14^{\circ}$ in EIT and $37^{\circ}$ in LASCO/C2 images. Likewise, average widths of structured CMEs with the axis perpendicular to the line of sight $(L)$ yielded greater values: $22^{\circ}$ in EIT and $58^{\circ}$ in LASCO/C2. The ratio $L / D$ was identical as measured in the field of view of both instruments, yielding a value of 1.6.

An elliptical cone model was developed in a later step, on the basis of the circular cone model by Zhao et al. (2002). It was applied to a set of full, bright, and front-sided halos, looking forward to investigate a relationship similar to $L / D$ in the fitted halos. The values representative for $D$ and $L$, the half-widths $\omega_{a}$ and $\omega_{b}$, yielded an average ratio $\left\langle\omega_{b} / \omega_{a}\right\rangle=1.11$, substantially different from the expected value of 1.6.

If indeed the source region lengths play a prime role for the configuration of CMEs, as suggested by Cremades \& Bothmer (2004), then it is expected that the source region characteristics for the set of analyzed halos should differ from those of the analyzed subset of structured CMEs. It is also important to know that the group of selected halos is most likely not representative of all CMEs, because the applied requirement of enhanced brightness implied attached selection effects, which might explain why most of these halo CMEs originated from compact, active regions. Finally, Cremades \& Bothmer (2004) found for the structured CMEs that the lengths of their associated source regions systematically increased with latitude. These extended source regions at higher latitudes do not seem to be typical sources of very bright full halos. Out of the ecliptic missions like the ESA Solar Orbiter or the NASA Solar Probe would allow for the first time to prove these associations.

\section{Acknowledgements}

This work is part of the scientific investigations of the project Stereo/Corona, in context of the International Max Planck Research School, supported by the German "Bundesministerium für Bildung und Forschung" through the "Deutsche Zentrum für Luftund Raumfahrt e.V." (DLR, German Space Agency) under project number 50 OC 0005. Stereo/Corona is a science and hardware contribution to the optical imaging package SECCHI currently being developed for the NASA STEREO mission to be launched in 2005. LASCO images used in this study are courtesy of the SOHO/LASCO consortium. $\mathrm{SOHO}$ is a project of international cooperation between ESA and NASA. The authors are thankful to Dr. Bernd Inhester and Rajat Thomas for their disinterested help.

\section{References}

Cremades, H., \& Bothmer, V. 2004, A\&A, 422, 307

Gibson, S. E., \& Low, B. C. 2000, J. Geophys. Res., 105, 18187

Hundhausen, A. J., Sawyer, C. B., House, L., Illing, R. M. E., \& Wagner, W. J. 1984, J. Geophys. Res. 89, 2639

Moran, T. G., \& Davila, J. M. 2004, Science, 305, 66

Munro, R. H., Gosling, J. T., Hildner, E., et al. 1979, Solar Phys., 61, 201

Trottet, G., \& MacQueen, R. M. 1980, Solar Phys., 68, 177

Webb, D. F. 1988, ApJ, 93, 1749

Zhao, X. P., Plunkett, S. P., \& Liu, W. 2002, J. Geophys. Res., 107, 10.1029/2001JA009143 


\section{Discussion}

Gopalswamy: I can understand that the filaments will not be seen well in white light when they are parallel to the limb. However, in the example you showed (SW limb), the filament (prominence) is also not seen well in EUV images, why is this?

Cremades: Well, that is the case for other events as well. Surprisingly, even though we don't see the filament in EIT, the configuration of the CME observed in C2 is still consistent with the of the orientation of the neutral line. In any case, it is generally more difficult to identify filaments (prominences) in EIT $195 \AA$ than in $\mathrm{H} \alpha$.

Koutchmy : Regarding the source region of the CME, did you pay attention to the filament length and strength as well, or did you pay attention only to the magnetic configuration?

CREmades: I also paid attention to the filament length (measured from Meudon and /or BBSO images). Neutral lines might be very large, but for the purpose of the measurement of the source region length, just the length of the erupting filament was the one taken into account. In the case of an active region in which no filament was observed, the length of the post-eruptive arcade of loops was considered as the source region length.

JIE ZHANG: The CME structure is related to the source region neutral line length and orientation, as you suggested. The question is, in many cases, the CME has a circular shape, but the source region is elongated. How can these be reconciled?

CRemades: Not only do source region length and orientation play a role, but also other factors such as location, ambient coronal configuration, etc. A long source region perpendicular to the limb, would yield a CME seen along its axis, with circular shape and overlying loops. But as this source region becomes more parallel to the line of the limb, the elongation of the filament should become more apparent. Concerning halos, the high degree of circularity (although there were ones with eccentricity ${ }^{-1}=1.3$ ) is for the moment attributed to the relatively short length of the source regions. 